\title{
Alternaria capsicicola sp. nov., a new species causing leaf spot of pepper (Capsicum
} annuum) in Malaysia

\begin{abstract}
A new species of Alternaria causing leaf spot of pepper (Capsicum annuum) obtained from the Cameron highlands, Pahang, Malaysia, was determined based on phylogenetic analyses, morphological characteristics, and pathogenicity assays. Phylogenetic analyses of combined dataset of the glyceraldehyde-3-phosphate dehydrogenase (gpd), Alternaria allergen a 1 (Alt a1) and calmodulin genes revealed that the new isolates clustered into a subclade distinct from the closely related Alternaria species A. tomato and A. burnsii. The solitary or short chains of conidia resemble those of A. burnsii. However, conidia with long beaks are morphologically similar to A. tomato. Hence, the pathogenic fungus is proposed as Alternaria capsicicola sp. nov. Pathogenicity assays indicated that A. capsicicola causes leaf spot on pepper.
\end{abstract}

Keyword: Morphological characteristics; Phylogenetic analyses; Systematics; Taxonomy 